\title{
THE TAUTOLOGICAL RING OF SPIN MODULI SPACES*
}

\author{
GILBERTO BINI
}

\begin{abstract}
We introduce the notion of tautological ring for the moduli space of spin curves. Moreover, we study some relations among tautological classes which are motivated by physics. Finally, we show that the Chow rings of these moduli spaces are tautological in low genus.
\end{abstract}

Key words. Moduli space, tautological ring, spin curves.

AMS subject classifications. 14H10, 14E08

1. Introduction. In the last decades, ideas set forth by physicists have revived a more detailed study of moduli spaces of curves. In particular, the contribution to the partition function for Polyakov's bosonic string is an integral [22], which can be reformulated on the moduli space $\mathcal{M}_{g}$ of genus $g$ smooth curves. To rewrite the integrand as a function, we need to know how to manipulate Chern classes on the moduli space. These classes are related to tautological classes, which have beeen defined by Mumford for $\overline{\mathcal{M}}_{g}$ - see [24]. This moduli space parametrizes stable curves, i.e., curves with a finite automorphism group, and contains $\mathcal{M}_{g}$ as a dense open set. Tautological classes are closed under cup product, so they form a ring, which is usually called the tautological ring [17].

Besides the bosonic string, physicists focus on the fermionic string as well. To deal with this theory, algebraic curves must be equipped with some extra structure. Following [10], we take into account the space which parametrizes curves with spin structures - see Section 2. Usually denoted by $\bar{S}_{g}$, this space is a branched covering of $\overline{\mathcal{M}}_{g}$ and compactifies the parameter space of pairs $(C, \eta)$, where $C$ is a genus $g$ algebraic curve and $\eta$ is a theta-characteristic, i.e., a square root of the canonical bundle. The space $\bar{S}_{g}$ is the union of two connected components parametrizing odd and even spin structures, respectively. Moreover, it is a $2^{2 g}$ degree finite covering of $\overline{\mathcal{M}}_{g}$. Expectedly, the contribution to fermionic strings should come from an integral on the moduli space of spin curves. If so, a natural problem is to study tautological classes and their intersection on $\bar{S}_{g}$. Unfortunately, the notion of tautological ring is missing.

In the present paper we introduce the tautological ring $\mathcal{R}^{\bullet}\left(\bar{S}_{g, n}\right)$ for the moduli space $\bar{S}_{g, n}$ of pointed spin curves. We show that it is a non-trivial extension of the image of the tautological ring of the moduli space of stable curves. To introduce $\mathcal{R} \bullet\left(\bar{S}_{g, n}\right)$, we need to describe the irreducible components of the boundary via some maps from other moduli spaces of spin curves. In some cases, these spaces are not of the form $\bar{S}_{g, n}$, but they are slightly modified as shown in Section 2. Motivated by physics, we come up with some relations among tautological classes which generalize those found by Mumford in connection with string theory [25]. We also give some examples of Chow ring in genus 3 and 4 . As a by-product we obtain that the rational Picard group of the open part is one dimensional. This matches with more general results proved in [23]. We end our paper with some speculation and open questions that come quite naturally from our research.

\footnotetext{
*Received November 24, 2008; accepted for publication September 7, 2009.

${ }^{\dagger}$ Dipartimento di Matematica, Università degli Studi di Milano, Via C. Saldini 50, 20133 Milano, Italy (gilberto.bini@unimi.it).
} 
2. Some basic facts on spin curves. In this section, we recall some basic definitions about spin structures. We closely follow [12], which we refer for more details. For a general approach see, for instance, [1].

Let $C$ be a Deligne-Mumford semistable curve and let $E$ be a complete, irreducible subcurve of $C$. The curve $E$ is said to be exceptional when it is smooth, rational, and intersects the other components in exactly two points. Moreover, $C$ is said to be quasi-stable when any two distinct exceptional components of $C$ are disjoint. In the sequel, $\tilde{C}$ will denote the subcurve $\overline{C \backslash \cup E_{i}}$ obtained from $C$ by removing all the exceptional components.

A spin curve of genus $g$ (see [12], $\S 2$ ) is the datum of a quasi-stable genus $g$ curve $C$ with an invertible sheaf $\zeta_{C}$ of degree $g-1$ on $C$ and a homomorphism of invertible sheaves $\alpha_{C}: \zeta_{C}^{\otimes 2} \longrightarrow \omega_{C}$ such that i) $\zeta_{C}$ has degree 1 on every exceptional component of $C$, and ii) $\alpha_{C}$ is not zero at a general point of every non-exceptional component of $C$. Therefore, $\alpha_{C}$ vanishes identically on all exceptional components of $C$ and induces an isomorphism $\tilde{\alpha}_{C}:\left.\zeta_{C}^{\otimes 2}\right|_{\tilde{C}} \longrightarrow \omega_{\tilde{C}}$. In particular, when $C$ is smooth, $\zeta_{C}$ is just a theta-characteristic on $C$. Two spin curves $\left(C, \zeta_{C}, \alpha_{C}\right)$ and $\left(C^{\prime}, \zeta_{C^{\prime}}, \alpha_{C^{\prime}}\right)$ are isomorphic if there are isomorphisms $\sigma: C \rightarrow C^{\prime}$ and $\tau: \sigma^{*}\left(\zeta_{C^{\prime}}\right) \rightarrow \zeta_{C}$ such that $\tau$ is compatible with the natural isomorphism between $\sigma^{*}\left(\omega_{C^{\prime}}\right)$ and $\omega_{C}$.

A family of spin curves is a flat family of quasi-stable curves $f: \mathcal{C} \rightarrow S$ with an invertible sheaf $\zeta_{f}$ on $\mathcal{C}$ and a homomorphism $\alpha_{f}: \zeta_{f}^{\otimes 2} \longrightarrow \omega_{f}$ such that the restriction of these data to any fiber of $f$ gives rise to a spin curve.

Two families of spin curves $f: \mathcal{C} \rightarrow S$ and $f^{\prime}: \mathcal{C}^{\prime} \rightarrow S$ are isomorphic if there are isomorphisms $\sigma: \mathcal{C} \longrightarrow \mathcal{C}^{\prime}$ and $\tau: \sigma^{*}\left(\zeta_{f^{\prime}}\right) \longrightarrow \zeta_{f}$ such that $f=f^{\prime} \circ \sigma$ and $\tau$ is compatible with the natural isomorphism between $\sigma^{*}\left(\omega_{f^{\prime}}\right)$ and $\omega_{f}$.

Let $\bar{S}_{g}$ be the moduli space of isomorphism classes of spin curves of genus $g$. Denote by $S_{g}$ the open subset consisting of classes of smooth curves. As shown in [12], $\S 5$, $\bar{S}_{g}$ has a natural structure of analytic orbifold given as follows. For any spin curve $Y$, there is a neighbourhood $U$ of $[Y]$ such that $U \cong B_{Y} / \operatorname{Aut}(Y)$, where $B_{Y}$ is a $3 g$-3-dimensional polydisk and $\operatorname{Aut}(Y)$ is the automorphism group of the spin curve $Y$. Alternatively, $\bar{S}_{g}$ may be viewed as a projective normal variety with finite quotient singularities.

The moduli space of spin curves can be slightly generalized as follows. For all integers $g, n, m_{1}, \ldots, m_{n}$, such that $2 g-2+n>0,0 \leq m_{i} \leq 1$ for every $i$, and $\sum_{i=1}^{n} m_{i}$ is even, we define

$$
\bar{S}_{g, n}^{\left(m_{1}, \ldots, m_{n}\right)}:=\left\{\left[\left(C, p_{1}, \ldots, p_{n} ; \zeta ; \alpha\right)\right]:\left(C, p_{1}, \ldots, p_{n}\right) \text { is a genus } g\right.
$$

quasi-stable projective curve with $n$ marked points;

$\zeta$ is a line bundle of degree $g-1+\frac{1}{2} \sum_{i=1}^{n} m_{i}$ on $C$

having degree 1 on every exceptional component of $C$, and

$\alpha: \zeta^{\otimes 2} \rightarrow \omega_{C}\left(\sum_{i=1}^{n} m_{i} p_{i}\right)$ is a homomorphism which

is not zero at a general point of every non-exceptional

component of $C\}$.

In order to put an analytic structure on $\bar{S}_{g, n}^{\left(m_{1}, \ldots, m_{n}\right)}$, we notice that Cornalba's 
construction in [12] can be easily adapted to $\bar{S}_{g, n}^{\left(m_{1}, \ldots, m_{n}\right)}$. Indeed, from the universal deformation of the stable model of $Y=\left(C, p_{1}, \ldots, p_{n}\right)$ we obtain exactly as in [12], $\S 4$, a universal deformation $\mathcal{U}_{Y} \rightarrow B_{Y}$ of $Y=\left(C, p_{1}, \ldots, p_{n} ; \zeta ; \alpha\right)$. Next, we put on $\bar{S}_{g, n}^{\left(m_{1}, \ldots, m_{n}\right)}$ the structure of the quotient analytic space $B_{Y} / \operatorname{Aut}(Y)$ following [12], $\S 5$. Alternatively, we can regard $\bar{S}_{g, n}^{\left(m_{1}, \ldots, m_{n}\right)}$ as the coarse moduli space associated to the stack of $r$-spin curves (in the easiest case $r=2$ ), which has been constructed by Jarvis in [20] and revisited by Abramovich and Jarvis in [1].

Analogously to $\bar{S}_{g}$ (see [12], Proposition 5.2), the spaces $\bar{S}_{g, n}^{\left(m_{1}, \ldots, m_{n}\right)}$ are normal projective varieties of complex dimension $3 g-3+n$. If $m_{1}=\ldots=m_{n}=0$, then $\bar{S}_{g, n}:=\bar{S}_{g, n}^{(0, \ldots, 0)}$ splits into two disjoint irreducible components $\bar{S}_{g, n}^{+}$and $\bar{S}_{g, n}^{-}$that consist of the even and the odd spin curves, respectively (see [12], Lemma 6.3).

The moduli space of spin curves comes equipped with a natural map $\nu$ : $\bar{S}_{g, n}^{\left(m_{1}, \ldots, m_{n}\right)} \rightarrow \overline{\mathcal{M}}_{g, n}$, which forgets the spin structure and stabilizes the domain curve. It is a finite morphism of degree $2^{2 g}$.

In general, if $P$ is a set with $n$ elements, it will be technically convenient to consider also $P$-pointed spin curves and $P$-pointed stable curves. These are simply spin curves (resp. stable curves) whose marked points are indexed by $P$ and not by $\{1, \ldots, n\}$. We shall denote by $\bar{S}_{g, P}^{\left(m_{1}, \ldots, m_{n}\right)}$ (resp. $\left.\overline{\mathcal{M}}_{g, P}\right)$ and by $S_{g, P}^{\left(m_{1}, \ldots, m_{n}\right)}$ the corresponding moduli spaces.

3. The tautological ring. In this section we first recall the definition of the tautological ring of $\overline{\mathcal{M}}_{g, P}$. Next, we introduce the tautological ring of the moduli space of spin curves.

For $p \in P$ denote by $\psi_{p}$ the universal cotangent class defined as follows. Let $\sigma_{p}: \overline{\mathcal{M}}_{g, P} \rightarrow \overline{\mathcal{M}}_{g, P \cup\{q\}}$ be the section of the forgetful morphism

$$
\phi: \overline{\mathcal{M}}_{g, P \cup\{q\}} \rightarrow \overline{\mathcal{M}}_{g, P} .
$$

Then set $\psi_{p}=c_{1}\left(\sigma_{p}^{*}\left(\omega_{\phi}\right)\right)$, where $\omega_{\phi}$ is the relative dualizing sheaf. For $A \subset P$ and $2 h-1+|A|>0,2 g-2 h-1+\left|A^{c}\right|>0$, recall the boundary maps (see, for instance, [3])

$$
\xi_{i r r}: \overline{\mathcal{M}}_{g-1, P \cup\left\{q_{1}, q_{2}\right\}} \rightarrow \overline{\mathcal{M}}_{g, P}
$$

and

$$
\xi_{h, A}: \overline{\mathcal{M}}_{h, A \cup\left\{r_{1}\right\}} \times \overline{\mathcal{M}}_{g-h, A^{c} \cup\left\{r_{2}\right\}} \rightarrow \overline{\mathcal{M}}_{g, P} .
$$

The tautological ring of $\overline{\mathcal{M}}_{g, P}$ is the smallest system of $\mathbb{Q}$-subalgebras of the cohomology rings which is closed under the morphisms (3.1), (3.2), (3.3) and contains the universal cotangent classes. We prefer to give the definition in terms of cohomology rings and not Chow rings for more generality.

We introduce some maps which will be used to define the tautological ring of $\bar{S}_{g, P}$. To begin with, we have the forgetful morphism

$$
\pi: \bar{S}_{g, P \cup\{q\}} \rightarrow \bar{S}_{g, P},
$$


where $|P|=n$. It forgets the point $q$ and stabilizes the spin curve. As remarked in [20], $\S 3.2, \bar{S}_{g, P \cup\{q\}}$ is isomorphic to the universal curve over $\bar{S}_{g, P}$. For $0 \leq i \leq g$ and $T \subset P$ with $2 i-1+|T|>0$ and $2 g-2 i-1+\left|T^{c}\right|>0$, let

$$
\begin{aligned}
& a_{i, T}^{+}: \bar{S}_{i, T \cup\left\{r_{1}\right\}}^{+} \times \bar{S}_{g-i, T^{c} \cup\left\{r_{2}\right\}}^{+} \rightarrow \bar{S}_{g, P}^{+}, \\
& b_{i, T}^{+}: \bar{S}_{i, T \cup\left\{r_{1}\right\}}^{-} \times \bar{S}_{g-i, T^{c} \cup\left\{r_{2}\right\}}^{-} \rightarrow \bar{S}_{g, P}^{+}, \\
& a_{i, T}^{-}: \bar{S}_{i, T \cup\left\{r_{1}\right\}}^{-} \times \bar{S}_{g-i, T^{c} \cup\left\{r_{2}\right\}}^{+} \rightarrow \bar{S}_{g, P}^{-}, \\
& b_{i, T}^{-}: \bar{S}_{i, T \cup\left\{r_{1}\right\}}^{+} \times \bar{S}_{g-i, T^{c} \cup\left\{r_{2}\right\}}^{-} \rightarrow \bar{S}_{g, P}^{-}
\end{aligned}
$$

be the following maps. A point in the domain is a pair of spin curves with the corresponding parity. A point in the image is obtained by attaching the two spin curves at a point and blowing-up. The exceptional $\mathbb{P}^{1}$ is equipped with the line bundle $\mathcal{O}_{\mathbb{P}^{1}}(1)$, which is glued to the other two characteristics of the spin curves. Along the lines of [12], the image set of these divisors are the boundary divisors $A_{i, T}^{+}, B_{i, T}^{+}, A_{i, T}^{-}, B_{i, T}^{-}$, respectively. For $i \geq 2$ and $i=1, T \neq \emptyset$, we set $\left[A_{i, T}^{ \pm}\right]=\alpha_{i, T}^{ \pm}$ and $\left[B_{i, T}^{ \pm}\right]=\beta_{i, T}^{ \pm}$, where $[\gamma]$ denotes the (rational) Poincaré dual of $\gamma$. For $i=1$ and $T=\emptyset$ we have $\alpha_{1, \emptyset}^{ \pm}=\frac{1}{2}\left[A_{1, \emptyset}^{ \pm}\right]$and $\beta_{1, \emptyset}^{ \pm}=\frac{1}{2}\left[B_{1, \emptyset}^{ \pm}\right]$because elliptic tails have an automorphism of order two. Notice that

$$
\alpha_{i, T}^{ \pm}=\alpha_{g-i, T^{c}}^{ \pm}, \quad \beta_{i, T}^{ \pm}=\beta_{g-i, T^{c}}^{ \pm}, \quad \alpha_{i, T}^{-}=\beta_{g-i, T^{c}}^{-} .
$$

Note in particular that $\beta_{[g / 2], \emptyset}^{-}=\alpha_{[g / 2], \emptyset}^{-}$. This justifies the assumption in [12] to disregard the class $\beta_{[g / 2], \emptyset}^{-}$.

The classes $\alpha_{i, T}^{ \pm}$and $\beta_{i, T}^{ \pm}$can be expressed as push-forwards under the maps (3.5), (3.6), (3.7), (3.8). Indeed, the general image point under these maps has an automorphism $\sigma$ of order two, which is given as follows. Pick $\left[C_{1}, p_{1}, \ldots, p_{|T|}, r_{1}, \mathcal{L}_{1}\right]$ and $\left[C_{2}, x_{1}, \ldots, x_{\left|T^{c}\right|}, r_{2}, \mathcal{L}_{2}\right]$. The automorphism $\sigma$ is the identity over the components of genus $i$ and $g-i$ and exchanges the two points - which can be assumed to be 0 and $\infty$ - on $\mathbb{P}^{1}$. Accordingly, the spin structure obtained by glueing $\left(\mathcal{L}_{1}\right)_{r_{1}}$ with $\mathcal{O}_{\mathbb{P}^{1}}(1)_{0}$ and $\left(\mathcal{L}_{2}\right)_{r_{2}}$ with $\mathcal{O}_{\mathbb{P}^{1}}(1)_{\infty}$ is sent to the spin structure obtained by glueing $\left(\mathcal{L}_{1}\right)_{r_{1}}$ with $\mathcal{O}_{\mathbb{P}^{1}}(1)_{\infty}$ and $\left(\mathcal{L}_{2}\right)_{r_{2}}$ with $\mathcal{O}_{\mathbb{P}^{1}}(1)_{0}$. Therefore, we set

$$
\alpha_{i, T}^{ \pm}=\frac{1}{2} a_{i, T, *}^{ \pm}(1), \quad \beta_{i, T}^{ \pm}=\frac{1}{2} b_{i, T, *}^{ \pm}(1) .
$$

We also have other natural maps, namely:

$$
\begin{aligned}
& a_{0}^{+}: \bar{S}_{g-1, P \cup\left\{q_{1}, q_{2}\right\}}^{(0, \ldots, 0,1,1)} \rightarrow \bar{S}_{g, P}^{+}, \\
& a_{0}^{-}: \bar{S}_{g-1, P \cup\left\{q_{1}, q_{2}\right\}}^{(0, \ldots, 0,1,1)} \rightarrow \bar{S}_{g, P}^{-}, \\
& b_{0}^{+}: \bar{S}_{g-1, P \cup\left\{m_{1}, m_{2}\right\}}^{+} \rightarrow \bar{S}_{g, P}^{-},
\end{aligned}
$$




$$
b_{0}^{-}: \bar{S}_{g-1, P \cup\left\{m_{1}, m_{2}\right\}}^{-} \rightarrow \bar{S}_{g, P}^{-} .
$$

The maps $a_{0}^{ \pm}$are defined as follows. Pick a point $\left[C, x_{1}, \ldots, x_{n}, q_{1}, q_{2}, \mathcal{L}\right]$ in the domain. As explained in [21], there are two possible identifications of the fibers $\mathcal{L}_{q_{1}}$ and $\mathcal{L}_{q_{2}}$. Each of them yields a spin structure on the curve which is obtained from $C$ by identifying $q_{1}$ and $q_{2}$. The first identification gives the map $a_{0}^{+}$and the second gives $a_{0}^{-}$. As for the maps $b_{0}^{ \pm}$, we identify $m_{1}$ and $m_{2}$ and we blow-up the resulting curve. Next, we glue $\mathcal{O}_{E}(1)$ on the exceptional component $E$ to the spin curve of the domain point. As before, we denote the closure of the images as $A_{0}^{ \pm}$and $B_{0}^{ \pm}$. The Poincaré duals are denoted by $\alpha_{0}^{ \pm}$and $\beta_{0}^{ \pm}$. We set $\alpha_{0}^{ \pm}=\frac{1}{2} a_{0, *}^{ \pm}(1)$ because the points $\left[C, p_{1}, \ldots, p_{n}, m_{1}, m_{2}, \mathcal{L}\right]$ and $\left[C, p_{1}, \ldots, p_{n}, m_{2}, m_{1}, \mathcal{L}\right]$ map to the same image point. On the other hand, we set $\beta_{0}^{ \pm}=\frac{1}{4} b_{0, *}^{ \pm}(1)$. In fact, we still have two points that map to the same one. For each of them, the generic member has an order two automorphism which is the identity on the genus $g-1$ component and exchanges two points - which can be assumed to be 0 and $\infty$-on the exceptional component. Accordingly, the spin structure obtained by glueing $\mathcal{L}_{m_{1}}$ with $\mathcal{O}_{\mathbb{P}^{1}}(1)_{0}$ and $\mathcal{L}_{m_{2}}$ with $\mathcal{O}_{\mathbb{P}^{1}}(1)_{\infty}$ is sent to the spin structure which is obtained by glueing $\mathcal{L}_{m_{1}}$ with $\mathcal{O}_{\mathbb{P}^{1}}(1)_{\infty}$ and $\mathcal{L}_{m_{2}}$ with $\mathcal{O}_{\mathbb{P}^{1}}(1)_{0}$.

The forgetful morphism has $n$ sections $\sigma_{p}: \bar{S}_{g, P} \rightarrow \bar{S}_{g, P \cup\{q\}}$. Let $\omega_{\pi}$ be the relative dualizing sheaf and define $\mathcal{L}_{p}:=\sigma_{p}^{*} \omega_{\pi}$. For each $p \in P$ set $\psi_{p}=c_{1}\left(\mathcal{L}_{p}\right)$.

Definition 3.1. The tautological ring $\mathcal{R} \bullet\left(\bar{S}_{g, P}\right)$ is the smallest system of $\mathbb{Q}$ subalgebras of the cohomology rings which is closed under the push-forwards of natural morphisms (3.4) through (3.12) and contains the classes $\psi_{p}$.

Let $i: S_{g, P} \rightarrow \bar{S}_{g, P}$. Then the tautological ring of $S_{g, P}$ is the image under $i^{*}$ of the tautological ring of $\bar{S}_{g, P}$.

The finite map $\nu: \bar{S}_{g, P} \rightarrow \overline{\mathcal{M}}_{g, P}$ induces an injective homomorphism $\nu^{*}$. We now prove that the image of $\mathcal{R} \bullet\left(\overline{\mathcal{M}}_{g, P}\right)$ lies in the tautological ring $\mathcal{R} \bullet\left(\bar{S}_{g, P}\right)$.

We recall the definition of push-forward of a map: see, for instance, [26], p. 178. If $\rho: V \rightarrow W$ is a morphism between two projective orbifolds, the push-forward $\rho_{*}$ is given by

$$
\rho_{*}(\alpha)=\mathcal{P}_{W}\left(\rho_{*} \mathcal{P}_{V}(\alpha)\right),
$$

where $\rho_{*}$ on the right hand side is the morphism induced in singular homology by $\rho$ and $\mathcal{P}_{A}(\gamma)$ is the (rational) Poincaré dual over the space $A$ of the cohomology class $\gamma$. The following result is probably known to experts, but we include the proof for the sake of completeness.

Lemma 3.2. Let $X, Y, X^{\prime}, Y^{\prime}$ be projective orbifolds. Let us consider the following diagram:

$$
\begin{array}{ccc}
X^{\prime} & \stackrel{\pi}{\rightarrow} & Y^{\prime} \\
\eta \downarrow & & \downarrow \nu \\
X & \rightarrow & Y, \\
& &
\end{array}
$$

where $\pi$ and $\phi$ are codimension one maps and $\eta$ and $\nu$ have finite degree. Then $\operatorname{deg}(\eta) \nu^{*} \phi_{*}=\operatorname{deg}(\nu) \pi_{*} \eta^{*}$.

Proof. Denote by $\alpha$ a class in $H^{*}(X)$ of degree $k$. By definition, the composition $\pi_{*} \eta^{*}$ is given by $\mathcal{P}_{Y^{\prime}}\left(\pi_{*} \mathcal{P}_{X^{\prime}}\left(\eta^{*} \alpha\right)\right)$. On the other hand, the class $\nu^{*} \phi_{*}(\alpha)$ is given by $\nu^{*} \mathcal{P}_{Y}\left(\phi_{*} \mathcal{P}_{X}(\alpha)\right)$. Notice that in homology we have: 


$$
\nu_{*} \pi_{*} \mathcal{P}_{X^{\prime}}\left(\eta^{*} \alpha\right)=\phi_{*} \eta_{*} \mathcal{P}_{X^{\prime}}\left(\eta^{*} \alpha\right)
$$

because the diagram (3.13) commutes. Let $\omega$ be a differential form of degree $k$ in $H^{*}(X)$. Then we have

$$
\begin{gathered}
\int_{\eta_{*} \mathcal{P}_{X^{\prime}}\left(\eta^{*}(\alpha)\right)} \omega=\int_{\mathcal{P}_{X^{\prime}}\left(\eta^{*} \alpha\right)} \eta^{*} \omega= \\
=\int_{X^{\prime}} \eta^{*} \alpha \wedge \eta^{*} \omega=\int_{X^{\prime}} \eta^{*}(\alpha \wedge \omega)=\operatorname{deg}(\eta) \int_{X} \alpha \wedge \omega .
\end{gathered}
$$

Hence we have

$$
\phi_{*} \eta_{*} \mathcal{P}_{X^{\prime}}\left(\eta^{*} \alpha\right)=\operatorname{deg}(\eta) \phi_{*} \mathcal{P}_{X}(\alpha) .
$$

Set $\beta=\pi_{*} \mathcal{P}_{X^{\prime}}\left(\eta^{*} \alpha\right)$ and $\gamma=\operatorname{deg}(\eta) \phi_{*} \mathcal{P}_{X}(\alpha)$. By (3.14) and (3.15) we have $\nu_{*}(\beta)=$ $\gamma$. On the other hand, we have

$$
\begin{aligned}
\int_{\gamma} \omega & =\int_{\nu_{*}(\beta)} \omega=\int_{\beta} \nu^{*} \omega=\int_{Y^{\prime}} \mathcal{P}_{Y^{\prime}}(\beta) \wedge \nu^{*} \omega \\
& =\int_{Y} \mathcal{P}_{Y}(\gamma) \wedge \omega=\frac{1}{\operatorname{deg}(\nu)} \int_{Y^{\prime}} \nu^{*} \mathcal{P}(\alpha) \wedge \nu^{*} \omega .
\end{aligned}
$$

This yields $\nu^{*} \mathcal{P}_{Y}(\gamma)=\operatorname{deg}(\nu) \mathcal{P}_{Y^{\prime}}(\beta)$. In other words, the following holds:

$$
\operatorname{deg}(\eta) \nu^{*} \mathcal{P}_{Y}\left(\phi_{*} \mathcal{P}_{X}(\alpha)\right)=\operatorname{deg}(\nu) \mathcal{P}_{Y^{\prime}}\left(\pi_{*} \mathcal{P}_{X^{\prime}}\left(\eta^{*} \alpha\right)\right),
$$

which proves the claim completely.

TheOrem 3.3. The tautological ring $\mathcal{R} \bullet\left(\overline{\mathcal{M}}_{g, P}\right)$ is a subring of $\mathcal{R} \bullet\left(\bar{S}_{g, P}^{+}\right)$(resp. $\left.\mathcal{R} \bullet\left(\bar{S}_{g, P}^{-}\right)\right)$.

Proof. First, notice that the following diagram commutes:

$$
\begin{array}{ccc}
\bar{S}_{g, P \cup\{q\}} & \stackrel{\pi}{\rightarrow} & \bar{S}_{g, P} \\
\eta \downarrow & & \downarrow \\
\overline{\mathcal{M}}_{g, P \cup\{q\}} & \underset{\phi}{\rightarrow} & \overline{\mathcal{M}}_{g, P} .
\end{array}
$$

The horizontal maps forget the point $q$ and the vertical arrows forget the spin structure on the curve. By Prop 2.5.1 in [13], we have $\eta^{*} \omega_{\phi} \cong \omega_{\pi}$. If $\tau_{p}$ and $\sigma_{p}$ are sections of $\phi$ and $\pi$ respectively, by the commutativity of (3.16) we also have that $\tau_{p} \nu=\eta \sigma_{p}$. Hence, we get

$$
\nu^{*} c_{1}\left(\tau_{p}^{*} \omega_{p}\right)=c_{1}\left(\sigma_{p}^{*} \omega_{\pi}\right) .
$$

Therefore, the image of the cotangent classes of $\overline{\mathcal{M}}_{g, P}$ is contained in $\mathcal{R} \bullet\left(\bar{S}_{g, P}\right)$. As for boundary maps, let us consider first the maps $\xi_{h, T}$. We deal with even spin curves because the odd case can be dealt with analogously. Let $X^{\prime}$ be the orbifold

$$
X^{\prime}=X_{1} \sqcup X_{2}=\bar{S}_{h, T \cup\left\{f_{1}\right\}}^{+} \times \bar{S}_{g-h, T^{c} \cup\left\{f_{2}\right\}}^{+} \sqcup \bar{S}_{h, T \cup\left\{r_{1}\right\}}^{-} \times \bar{S}_{g-h, T^{c} \cup\left\{r_{2}\right\}}^{-} .
$$


We have a diagram as follows:

$$
\begin{array}{rll}
X_{1} \sqcup X_{2} & \longrightarrow & \bar{S}_{g, P}^{+} \\
u \downarrow \downarrow v & & \downarrow \nu \\
\overline{\mathcal{M}}_{h, T \cup\left\{l_{1}\right\}} \times \overline{\mathcal{M}}_{g-h, T^{c} \cup\left\{l_{2}\right\}} & \longrightarrow \overline{\mathcal{M}}_{g, P}
\end{array}
$$

where the upper horizontal maps are $a_{h, T}^{+}$and $a_{h, T}^{-}$, respectively. The vertical arrows forget the corresponding spin structure. Denote by $\eta$ the map $(u, v)$ and by $\pi$ the map $\left(a_{h, T}^{+}, a_{h, T}^{-}\right)$. Analogously to Lemma 3.13 , we have

$$
\nu^{*} \xi_{h, T, *}=a_{h, T, *}^{+} u^{*}+a_{h, T, *}^{-} v^{*}
$$

since $\operatorname{deg}(\nu)=\operatorname{deg}(u)+\operatorname{deg}(v)$.

As for the other boundary maps, the even and the odd case has to be dealt with simultaneously. More precisely, let

$$
Y_{1} \sqcup Y_{2} \sqcup Y=\bar{S}_{g-1, P \cup\left\{a_{1}, a_{2}\right\}}^{+} \sqcup \bar{S}_{g-1, P \cup\left\{b_{1}, b_{2}\right\}}^{-} \sqcup \bar{S}_{g-1, P \cup\left\{c_{1}, c_{2}\right\}}^{(0,0, \ldots, 1,1)} .
$$

There exist maps from this disjoint union to $\bar{S}_{g, P}$ which are given by $a_{0}^{ \pm} b_{0}^{ \pm}$. We thus have a diagram

$$
\begin{array}{rll}
Y_{1} \sqcup Y_{2} \sqcup Y & \longrightarrow & \bar{S}_{g, P}^{+} \\
\tau \downarrow \downarrow \downarrow \downarrow \mu & & \downarrow \nu \\
\overline{\mathcal{M}}_{g-1, P \cup\{q, t\}} & \underset{\xi_{i r r}}{\rightarrow} & \overline{\mathcal{M}}_{g, P},
\end{array}
$$

where the upper horizontal map is given by $\left(b_{0}^{+}, b_{0}^{-}, a_{0}^{+}, a_{0}^{-}\right)$. The vertical arrows forget the corresponding spin structure. We notice that the map $\xi_{i r r}$ has degree two so Lemma 3.2 must be slightlty modified. However, it is easy to prove that the following holds:

$$
\begin{aligned}
& 2(\operatorname{deg}(\tau)+\operatorname{deg}(\xi)+\operatorname{deg}(\mu)) \nu^{*} \xi_{i r r, *} \\
& =\operatorname{deg}(\nu)\left(b_{0, *}^{+} \tau^{*}+b_{0, *}^{-} \xi^{*}+a_{0, *}^{+} \mu^{*}+a_{0, *}^{-} \mu^{*}\right) .
\end{aligned}
$$

Let $Q \in H^{*}\left(\overline{\mathcal{M}}_{g, P \cup\{q\}}\right)$ be a polynomial in the class $\psi_{p}$. If we apply Lemma 3.2 or (3.17) and (3.18), we see that the image of $\phi_{*}(Q)$ is a class in $\mathcal{R} \bullet\left(\bar{S}_{g, P}\right)$.

REMARK 3.4. Notice that

$$
\begin{gathered}
\nu^{*}\left(\xi_{h, A, *}(1)\right)=a_{h, A, *}^{ \pm}(1)+b_{h, A, *}^{ \pm}(1)=2 \alpha_{h, A}^{ \pm}+2 \beta_{h, A}^{ \pm}, \\
\nu^{*}\left(\frac{\xi_{i r r, *}(1)}{2}\right)=\frac{a_{0, *}^{ \pm}(1)}{2}+\frac{b_{0, *}^{ \pm}(1)}{2}=\alpha_{0}^{ \pm}+2 \beta_{0}^{ \pm} .
\end{gathered}
$$
$\bar{S}_{g}$

We thus obtain a generalization of the formulas given in [12] for the moduli space 
4. Some tautological classes and their relations. In this section we present some tautological classes and study some relations among them.

Let us recall the definition of the Mumford-Morita-Miller classes on $\overline{\mathcal{M}}_{g, P}$. Set

$$
\widetilde{\kappa}_{m}=\phi_{*}\left(c_{1}\left(\omega_{\phi}\right)^{m+1}\right) .
$$

Analogously, define the classes - by abuse of notation we still denote them by the same letter -

$$
\widetilde{\kappa}_{m}=\pi_{*}\left(c_{1}\left(\omega_{\pi}\right)^{m+1}\right) .
$$

By Diagram 3.16 and $\eta^{*} \omega_{\phi}=\omega_{\pi}$ we have $\nu^{*}\left(\widetilde{\kappa}_{m}\right)=\widetilde{\kappa}_{m}$.

Let us consider the sheaf $\pi_{*} \omega_{\pi}^{n}$ for $n \geq 1$ on $\bar{S}_{g, P}$. By base change applied to Diagram 3.16, we have $\pi_{*} \omega_{\pi}^{n}=\nu^{*} \phi_{*} \omega_{\phi}^{n}$. By abuse of notation, we denote by the same symbol the pull-back under $\nu$ of the Chern classes of $\phi_{*} \omega_{\phi}^{n}$. Note in particular that $\nu^{*}(\lambda)=\lambda$, where $\lambda$ is the first Chern class of the Hodge bundle.

Let $\mu$ denote the class of the line bundle $\operatorname{det} R f_{*} \zeta_{f}$, where $\zeta_{f}$ is the line bundle of degree $g-1$ on a family of spin curves $f: \mathcal{X} \rightarrow B$. The class $\mu$ is tautological since $\alpha_{0}=4 \lambda+8 \mu-$ see $[11]$.

Proposition 4.1. For $g \geq 1$ the ring $\mathcal{R} \bullet\left(\bar{S}_{g, P}\right)$ is a non-trivial extension of $\nu^{*}\left(\mathcal{R} \bullet\left(\overline{\mathcal{M}}_{g, P}\right)\right)$.

Proof. It suffices to show there exist non-zero elements which belong to $\mathcal{R}^{\bullet}\left(\bar{S}_{g, P}\right) \backslash \nu^{*} \mathcal{R} \bullet\left(\overline{\mathcal{M}}_{g, P}\right)$. Let us consider the even case. The odd case can be dealt with analogously. On the contrary, suppose $\alpha_{0}^{+}=\nu^{*}(t)$ for some $t$. Since $\alpha_{0}^{+} \in P i c_{\mathbb{Q}}\left(\bar{S}_{g, P}\right)$, the class $t$ belongs to $P i c_{\mathbb{Q}}\left(\overline{\mathcal{M}}_{g, P}\right)$, i.e., $t=a \lambda+\sum_{i} b_{i} \delta_{i}$. By pull-back we have

$$
\alpha_{0}^{+}=a \lambda^{+}+b_{0}\left(\alpha_{0}^{+}+2 \beta_{0}^{+}\right)+\sum b_{i}\left(2 \alpha_{i}^{+}+2 \beta_{i}^{+}\right) .
$$

Since the classes $\lambda^{+}, \alpha_{i}^{+}$are linearly independent, we get $b_{0}=1$ and $b_{0}=0$, which is clearly impossible.

Motivated from physics, we express the Chern classes of $\mathbb{W}_{b}=\pi_{*} \omega_{\pi}^{b}$ for $b \geq 2$ on $\bar{S}_{g, P}$ in terms of those of $\mathbb{W}_{1}$. This can be done again by applying the GrothendieckRiemann-Roch Theorem. We recall some conventional notation on Bernoulli numbers and Bernoulli polynomials. Their definition is given via the following identities:

$$
\begin{gathered}
\frac{x}{e^{x}-1}=\sum_{j \geq 1} B_{j} \frac{x^{j}}{j !}, \\
e^{u x} \frac{x}{e^{x}-1}=\sum_{n \geq 0} B_{n}(u) \frac{x^{n}}{n !} .
\end{gathered}
$$

where $x$ and $u$ are formal variables.

Proposition 4.2. The following hold:

i)

$$
\operatorname{ch}_{2 d}\left(\mathbb{W}_{b}\right)=\left\{\begin{array}{cc}
0 & b=1, \\
\frac{B_{2 d+1}(b)}{(2 d+1) !} \widetilde{\kappa}_{2 d} & \text { otherwise. }
\end{array}\right.
$$


ii)

$$
c h_{2 d-1}\left(\mathbb{W}_{b}\right)=\frac{B_{2 d}(b)}{B_{2 d}} \operatorname{ch}\left(\mathbb{W}_{1}\right)+\left(1-\frac{B_{2 d}(b)}{B_{2 d}}\right) \Delta_{2 d-1},
$$

where

$$
\begin{aligned}
\Delta_{2 d-1} & =\frac{1}{2} \frac{B_{2 d}}{(2 d) !}\left\{a_{0, *}^{ \pm}\left(\psi_{q_{1}}^{2 a-2}-\psi_{q_{1}}^{2 a-3} \psi_{q_{2}}+\ldots+\psi_{q_{2}}^{2 a-2}\right)\right. \\
& \left.+b_{0, *}^{ \pm}\left(\psi_{q_{1}}^{2 a-2}-\psi_{q_{1}}^{2 a-3} \psi_{q_{2}}+\ldots+\psi_{q_{2}}^{2 a-2}\right)\right\} \\
& +\frac{1}{2} \frac{B_{2 d}}{(2 d) !} \sum_{h=0}^{g} \sum_{T \subset P}\left\{a_{h, T, *}^{ \pm}\left(\psi_{r_{1}}^{2 a-2} \otimes 1-\psi_{r_{1}}^{2 a-3} \otimes \psi_{r_{2}}+\ldots+1 \otimes \psi_{r_{2}}^{2 a-2}\right)\right. \\
& \left.+b_{h, T, *}^{ \pm}\left(\psi_{r_{1}}^{2 a-2} \otimes 1-\psi_{r_{1}}^{2 a-3} \otimes \psi_{r_{2}}+\ldots+1 \otimes \psi_{r_{2}}^{2 a-2}\right)\right\}
\end{aligned}
$$

Proof. By [24] and [7], i) follows easily by pull-back under $\nu$.

ii) Set $\omega_{\phi}^{b}=\mathbb{E}_{b}$. By base change with respect to the map $\nu$ in (3.13), we have $\pi_{*} \eta^{*} \mathbb{E}_{b}=\nu^{*} \phi_{*} \mathbb{E}_{b}$. As for Chern classes we have $c_{j}\left(\mathbb{W}_{b}\right)=c_{j}\left(\pi_{*} \eta^{*} \mathbb{E}_{b}\right)=\nu^{*} c_{j}\left(\phi_{*} \mathbb{E}_{b}\right)$. By [7], we have

$$
c h_{2 a-1}\left(\mathbb{E}_{b}\right)=\frac{B_{2 a}(b)}{(2 a) !} \widetilde{\kappa}_{2 a-1}+\delta_{2 a-1}
$$

where

$$
\begin{aligned}
\delta_{2 a-1} & =\frac{B_{2 a}}{(2 a) !}\left\{\frac{1}{2} \xi_{i r r}, *\left(\psi_{q_{1}}^{2 a-2}-\psi_{q_{1}}^{2 a-3} \psi_{q_{2}}+\ldots+\psi_{q_{2}}^{2 a-2}\right)\right. \\
& \left.+\frac{1}{2} \sum_{h=0}^{g} \sum_{T \subset P} \xi_{h, T, *}\left(\psi_{r_{1}}^{2 a-2} \otimes 1-\psi_{r_{1}}^{2 a-3} \otimes \psi_{r_{2}}+\ldots+1 \otimes \psi_{r_{2}}^{2 a-2}\right)\right\} .
\end{aligned}
$$

By [24], we get

$$
\widetilde{\kappa}_{2 a-1}=\frac{(2 a) !}{B_{2 a}}\left(\operatorname{ch}_{2 a-1}\left(\mathbb{E}_{1}\right)-\delta_{2 a-1}\right) .
$$

Thus, we have

$$
c h_{2 a-1}\left(\mathbb{E}_{b}\right)=\frac{B_{2 a}(b)}{B_{2 a}} \operatorname{ch}_{2 a-1}\left(\mathbb{E}_{1}\right)+\left(1-\frac{B_{2 a}(b)}{B_{2 a}}\right) \delta_{2 a-1} .
$$

As remarked above, the Chern character of $\mathbb{E}_{b}$ on $\bar{S}_{g, P}$ is the pull-back under $\nu$ of the Chern character of $\mathbb{E}_{b}$ on $\overline{\mathcal{M}}_{g, P}$. To prove the claim we just need to compute $\nu^{*}\left(\delta_{2 a-1}\right)$. The claim follows from Theorem 3.3.

REMARK 4.3. For $a=1$ we get

$$
c_{1}\left(\mathbb{W}_{b}\right)=\left(6 b^{2}-6 b+1\right) \lambda-\left(\begin{array}{l}
b \\
2
\end{array}\right)\left(2 \sum_{i=1}^{\lfloor g / 2\rfloor}\left(\alpha_{i}+\beta_{i}\right)+\alpha_{0}+2 \beta_{0}\right),
$$

which is the pull-back on $\bar{S}_{g, P}$ of the relation obtained by Mumford in [25]. 
Other relations between tautological classes may be obtained by applying the Grothendieck-Riemann-Roch Theorem to the universal root of the line bundle $\omega_{\pi}^{s}$ and its generalizations. This has been done in [14]. For the case at hand, the formulas in [14] read as follows. Let $\mathcal{S}$ be the universal root of the bundle $\omega_{\pi}^{s}$. Then the following holds [14], Thm. 1.1.1:

$$
\operatorname{ch}\left(R^{\bullet} \pi_{*} \mathcal{S}\right)=\sum_{d \geq 0}\left(\frac{B_{d+1}(s / 2)}{(d+1) !} \widetilde{\kappa}_{d}+\frac{1}{2} \sum_{q=0}^{1} \frac{2 B_{d+1}(q / 2)}{(d+1) !}\left(j_{q}\right)_{*}\left(\gamma_{d-1}\right)\right),
$$

where

$$
j_{0}=\sum_{i, T}\left(a_{i, T}^{ \pm}\right)+\left(b_{i, T}^{ \pm}\right)+a_{0}^{ \pm}, \quad j_{1}=b_{0}^{ \pm},
$$

and $\gamma_{d-1}=\sum_{i+j=d}(-\psi)^{i}\left(\widehat{\psi}^{j}\right)$, where $\psi$ and $\widehat{\psi}$ are the first Chern classes of the line bundles whose fibers are the cotangent line to the first branch and the cotangent line to the second branch of the singular locus.

5. Some tautological rings in low genus. In this section we study some tautological rings in low genus. More specifically, we determine the Chow rings of some moduli spaces of spin structure and show they are tautological. To begin with, we handle some cases in genus one.

Lemma 5.1. The degree three covering

$$
\gamma: \bar{S}_{1,1}^{+} \rightarrow \overline{\mathcal{M}}_{1,1}=\mathcal{M}_{1,1} \cup\left\{\Delta_{0}\right\} \cong \mathbb{P}^{1}
$$

is branched at $\Delta_{0}$ and $[E, 0]$, where $\operatorname{Aut}(E) \cong \mathbb{Z} / m \mathbb{Z}$ for $m=4,6$ and $\Delta_{0}$ is the class of the irreducible nodal curve.

Proof. The divisor $\Delta_{0}$ is a branch point of $\gamma$ since it corresponds to an irreducible nodal curve. Let $p_{1}=0, p_{2}, p_{3}$ and $p_{4}$ be the two-torsion points of $E$. The even theta-characteristics are given by $\mathcal{O}_{E}\left(p_{1}-p_{j}\right)$ for $j \in\{2,3,4\}$. If $\operatorname{Aut}(E) \cong \mathbb{Z} / 4 \mathbb{Z}$, the torsion points are given by $0,1 / 2, \sqrt{-1} / 2,1 / 2+\sqrt{-1} / 2$. The generator of Aut $(E)$ exchanges the two theta-characteristics $\mathcal{O}_{E}\left(0-\frac{1}{2}\right)$ and $\mathcal{O}_{E}\left(0-\frac{\sqrt{-1}}{2}\right)$ and fixes the remaining theta characteristic. Thus, the map $\gamma$ is branched at the point in $\overline{\mathcal{M}}_{1,1}$ corresponding to this curve. Analogously, the elliptic curve with $\operatorname{Aut}(E) \cong \mathbb{Z} / 6 \mathbb{Z}$ yields a total ramification point of $\gamma$. The variety $\bar{S}_{1,1}^{+}$is smooth since it is normal and has dimension one. Its genus is zero because the following holds:

$$
2-2 g\left(\bar{S}_{1,1}^{+}\right)=6-2-1-1=2 .
$$

In other words, $\bar{S}_{1,1}^{+} \cong \mathbb{P}^{1}$.

THEOREM 5.2. The following isomorphisms hold: i)

$$
\begin{gathered}
\left.H^{*}\left(\bar{S}_{1,1}^{-}\right) \cong \mathbb{Q}\left[\alpha_{0}^{-}\right] /\left(\left(\alpha_{0}^{-}\right)^{2}\right)\right), \\
H^{*}\left(\bar{S}_{1,2}^{-}\right) \cong \mathbb{Q}\left[\alpha_{0}^{-}, \alpha_{1}^{-}\right] /\left(\left(\alpha_{0}^{-}\right)^{2}, 12\left(\alpha_{1}^{-}\right)^{2}+\left(\alpha_{0}^{-}\right)\left(\alpha_{1}^{-}\right)\right),
\end{gathered}
$$


where $\alpha_{1}^{-}=\alpha_{1, \emptyset}^{-}$

ii)

$$
\begin{gathered}
H^{*}\left(\bar{S}_{1,1}^{+}\right) \cong \mathbb{Q}\left[\alpha_{0}^{+}\right] /\left(\left(\alpha_{0}^{+}\right)^{2}\right), \\
H^{*}\left(\bar{S}_{1,2}^{+}\right) \cong \mathbb{Q}\left[\alpha_{0}^{+}, \alpha_{1}^{+}\right] /\left(\left(\alpha_{0}^{+}\right)^{2}, 8\left(\alpha_{1}^{+}\right)^{2}+\left(\alpha_{0}^{+}\right)\left(\alpha_{1}^{+}\right)\right),
\end{gathered}
$$

where $\alpha_{1}^{+}=\alpha_{1, \emptyset}^{+}$.

Proof. i) We recall that there exists only one odd theta-characteristic on a genus one curve. In other words, $\bar{S}_{1,1}^{-} \cong \overline{\mathcal{M}}_{1,1}$ and $\bar{S}_{1,2}^{-} \cong \overline{\mathcal{M}}_{1,2}$. Thus, the claim follows: see, e.g., [9].

ii) By Lemma 5.1, the space $\bar{S}_{1,1}^{+}$is isomorphic to $\mathbb{P}^{1}$. Since $\gamma^{*}\left(\delta_{i r r}\right)=3 \alpha_{0}^{+}$, we have $\left(\alpha_{0}^{+}\right)^{2}=0$. As for $\bar{S}_{1,2}^{+}$, let $\delta_{i r r}$ and $\delta_{1}$ be the (Poincaré) duals of the boundary divisors on $\overline{\mathcal{M}}_{1,2}$. We recall that $\delta_{i r r}^{2}=0$ and $\delta_{1}^{2}+\frac{1}{12} \delta_{i r r} \delta_{1}=0$. Moreover, if $\gamma_{1,2}^{+}: \bar{S}_{1,2}^{+} \rightarrow \overline{\mathcal{M}}_{1,2}$ is the $3: 1$ covering of $\overline{\mathcal{M}}_{1,2}$, then we have:

$$
\left(\gamma_{1,2}^{+}\right)^{*}\left(\delta_{i r r}\right)=3 \alpha_{0}^{+}, \quad\left(\gamma_{1,2}^{+}\right)^{*}\left(\delta_{1}\right)=2 \alpha_{1}^{+} .
$$

Hence, the claim follows.

As proved in [5], the cohomology ring of $\bar{S}_{2}^{ \pm}$is tautological. Here we deal with the moduli spaces $\bar{S}_{2,\{p\}}^{ \pm}$.

Proposition 5.3. $S_{2,\{p\}}^{ \pm}$is the union of two affine subvarieties:

$$
U_{0}^{ \pm}=\{[C, p, \mathcal{L}]: p \text { is not a Weierstrass point of } C\}
$$

and

$$
U_{1}^{ \pm}=\{[C, p, \mathcal{L}]: p \text { is a Weierstrass point of } C\} .
$$

Each of them has the Chow ring of a point; hence the Chow ring of $S_{2,\{p\}}^{ \pm}$is generated by $\left[S_{2,\{p\}}^{ \pm}\right]$and $\left[U_{1}^{ \pm}\right]$.

Proof. The moduli space $\mathcal{M}_{2,1}$ is the union of two affine subvarieties, each of them parametrizing pairs $(C, p)$ where the marked point is a Weierstrass point or not. $U_{0}^{ \pm}$ and $U_{1}^{ \pm}$are the preimages under a finite morphism of these two subvarieties; hence they are affine. Let us consider the following morphisms:

$$
\begin{aligned}
& f^{+}: \mathcal{M}_{0,7} \rightarrow U_{0}^{+}, \\
& f^{-}: \mathcal{M}_{0,7} \rightarrow U_{0}^{-}, \\
& g^{+}: \mathcal{M}_{0,6} \rightarrow U_{1}^{+}, \\
& g^{-}: \mathcal{M}_{0,6} \rightarrow U_{1}^{-} .
\end{aligned}
$$


The morphism $f^{+}$maps the rational curve $\left[\mathbb{P}^{1} ; p_{1}, \ldots, p_{6}, q\right]$ to the genus two curve $C$ (covering of $\mathbb{P}^{1}$ ) with branch points $p_{1}, \ldots, p_{6}$ and with marked point one of the two points in the preimage of $q$. The spin structure is given by $\mathcal{O}_{C}\left(r_{1}\right)$, where $r_{i}$ is the preimage of $p_{i}$. The morphism $f^{-}$is defined in a similar way. The spin structure is given by $\mathcal{O}_{C}\left(r_{1}+r_{2}-r_{3}\right)$.

As for the morphisms, $g^{ \pm}$we map the rational curve $\left[\mathbb{P}^{1} ; p_{1}, \ldots, p_{6}\right]$ to $\left[C, r_{6}, \mathcal{L}\right]$, where $C$ is as above and $\mathcal{L}$ is $\mathcal{O}_{C}\left(r_{1}\right)$ for $g^{+}$and $\mathcal{O}_{C}\left(r_{1}+r_{2}-r_{3}\right)$ for $g^{-}$. The existence of these maps show that $U_{0}^{ \pm}$and $U_{1}^{ \pm}$have the Chow ring of a point. Hence the claim follows.

COROLlary 5.4. The rational Chow ring of $S_{2,\{p\}}^{ \pm}$is isomorphic to $\mathbb{Q}\left[\psi_{p}^{ \pm}\right] /\left(\left(\psi_{p}^{ \pm}\right)^{2}\right)$. In particular, it is tautological.

Proof. As proved in [15], Thm. 2.2, the class $\psi_{p}$ is a multiple of the class $[U]$ on $\mathcal{M}_{2,\{p\}}$, where $U$ is the locus of curves where the marked point is a Weierstrass point. This means that $\psi_{p}^{ \pm}$is a multiple of $\left[U_{1}^{ \pm}\right]$. In other words, the natural morphism

$$
\mathbb{Q}\left[\psi_{p}^{ \pm}\right] /\left(\left(\psi_{p}^{ \pm}\right)^{2}\right) \rightarrow A_{\mathbb{Q}}\left(S_{2,\{p\}}^{ \pm}\right)
$$

is both injective and surjective.

THEOREM 5.5. The cohomology groups of $\bar{S}_{2,1}^{ \pm}$are generated by tautological classes.

Proof. First, we notice that the maps (5.1) and (5.2) define a surjective morphisms

$$
f^{ \pm}: \overline{\mathcal{M}}_{0,7} \rightarrow \bar{S}_{2,1}^{ \pm} \text {. }
$$

In fact, $\overline{\mathcal{M}}_{0,7}$ is normal and $\bar{S}_{2,1}^{ \pm}$is finite over $\overline{\mathcal{M}}_{2,1}$. This means that $H^{k}\left(\bar{S}_{2,1}^{ \pm}\right) \subset$ $H^{k}\left(\overline{\mathcal{M}}_{0,7}\right)$. In particular, the odd cohomology vanishes and the even cohomology is algebraic. We also have that $h^{2,0}\left(\bar{S}_{2,1}^{ \pm}\right)=0$. By the exact sequence associated to the exponential sequence, this implies that $H^{2}\left(\bar{S}_{2,1}^{ \pm}\right)$is isomorphic to the rational Picard group, which is isomorphic to $A_{3}\left(\bar{S}_{2,1}^{ \pm}\right)$because the singularities are of finite quotient type. By the exact sequence

$$
A_{k}\left(\partial S_{2,1}^{ \pm}\right) \rightarrow A_{k}\left(\bar{S}_{2,1}^{ \pm}\right) \rightarrow A_{k}\left(S_{2,1}^{ \pm}\right) \rightarrow 0,
$$

the Picard group is generated by tautological classes; hence $H^{2}$ is tautological. By the Hard Lefschetz Theorem, the $H^{6}$ is isomorphic to $H^{2}$ and so is tautological as well. In fact, an isomorphism is given via multiplication by $\kappa_{1}^{2}$. We recall that $\kappa_{1}$ is ample since it is the pull-back under a finite map of an ample class on $\overline{\mathcal{M}}_{2,1}$. As for the codimension two cycles, by Corollary 5.4 we have

$$
A_{2}\left(\bar{S}_{2,1}^{ \pm}-S_{2,1}^{ \pm}\right) \rightarrow A_{2}\left(\bar{S}_{2,1}^{ \pm}\right) \rightarrow 0
$$

By iterating the exact sequence, we have

$$
A_{2}\left(\partial\left(\bar{S}_{2,1}^{ \pm}-S_{2,1}^{ \pm}\right)\right) \rightarrow A_{2}\left(\bar{S}_{2,1}^{ \pm}-S_{2,1}^{ \pm}\right) \rightarrow A_{2}\left(\operatorname{Int}\left(\bar{S}_{2,1}^{ \pm}-S_{2,1}^{ \pm}\right)\right) \rightarrow 0 .
$$

The space $\operatorname{Int}\left(\bar{S}_{2,1}^{ \pm}-S_{2,1}^{ \pm}\right)$is given by the images of the maps - restricted to the open part - which are defined in Section 1, namely:

$$
a_{0}^{ \pm}: S_{1,3}^{(0,1,1)} \rightarrow \operatorname{Int}\left(\Delta_{0}^{ \pm}\right)
$$




$$
\begin{gathered}
b_{0}^{ \pm}: S_{1,3} \rightarrow \operatorname{Int}\left(\Delta_{1}^{ \pm}\right), \\
a_{1}^{+}: S_{1,1}^{+} \times S_{1,2}^{+} \rightarrow \operatorname{Int}\left(\Delta_{2}^{+}\right), \\
b_{1}^{+}: S_{1,1}^{-} \times S_{1,2}^{-} \rightarrow \operatorname{Int}\left(\Gamma_{2}^{+}\right), \\
a_{1}^{-}: S_{1,1}^{+} \times S_{1,2}^{+} \rightarrow \operatorname{Int}\left(\Delta_{2}^{-}\right), \\
b_{1}^{-}: S_{1,1}^{+} \times S_{1,2}^{-} \rightarrow \operatorname{Int}\left(\Gamma_{2}^{-}\right),
\end{gathered}
$$

As proved in Theorem 5.2, the cohomology of $\bar{S}_{1,1}^{ \pm}$and $\bar{S}_{1,2}^{ \pm}$is generated by boundary classes, so the codimension two Chow group of these components is trivial. As for the other components, notice we have the following maps:

$$
\begin{gathered}
u: \mathcal{M}_{0,6} \rightarrow S_{1,3}^{ \pm}, \\
v: \mathcal{M}_{0,6} \rightarrow S_{1,3}^{(0,1,1)} .
\end{gathered}
$$

The map $u$ sends $\left[\mathbb{P}^{1}, p_{1}, \ldots, p_{4}, q_{1}, q_{2}\right]$ to the genus one curve $C$ branched over $p_{1}, \ldots, p_{4}$ with ramification point $r_{i}$ for $i=1,2,3,4$. Fix a point $x_{1}$ over $q_{1}$ and mark the two points $y_{1}$ and $y_{2}$ in the preimage of $q_{2}$. Thus, $\left(C, x_{1}, y_{1}, y_{2}\right)$ is a genus one curve with three marked points. Notice that another choice of the marked points over $q_{1}$ and $q_{2}$ yields an isomorphic curve. If we choose $\mathcal{O}_{C}\left(r_{1}-r_{2}\right)$, we have an even spin curve. This is the image of the map $u$.

As for the map $v$, consider the moduli point $\left[\mathbb{P}^{1}, p_{1}, \ldots, p_{4}, q_{1}, q_{2}\right]$. As before, we have a curve $F$. We also mark the two points in the preimage of $q_{1}$ and a point in the preimage of $q_{2}$. We associate the spin structure $\mathcal{O}_{F}\left(r_{1}\right)$ with this curve.

In both cases, we deduce that the codimension two Chow ring of the images of $a_{0}^{ \pm}$and $b_{0}^{ \pm}$is trivial since $A^{k}\left(\mathcal{M}_{0, n}\right)$ is trivial for $k>0$.

Now, the exact sequence 5.4 becomes

$$
A_{2}\left(\partial\left(\bar{S}_{2,1}^{ \pm}-S_{2,1}^{ \pm}\right)\right) \rightarrow A_{2}\left(\bar{S}_{2,1}^{ \pm}-S_{2,1}^{ \pm}\right) \rightarrow 0 .
$$

Notice that $B:=\partial\left(\bar{S}_{2,1}^{ \pm}-S_{2,1}^{ \pm}\right)$is two-dimensional. By iterating the exact sequence above, we get

$$
0 \rightarrow A_{2}(B) \rightarrow A_{2}(\operatorname{Int}(B)) \rightarrow 0,
$$

since $B-\operatorname{Int}(B)$ is one-dimensional. The $\operatorname{ring} A_{2}(\operatorname{Int}(B))$ is generated by components which are dominated by products of moduli spaces of the form $S_{1, n}^{ \pm} \times S_{0, m} \times S_{0, k}^{(0,0,1, \ldots, 1)}$ for $n \leq 2$. The cohomology of these spaces is tautological by Lemma 5.2 and by the isomorphisms $S_{0, m} \cong \mathcal{M}_{0, m}, \mathcal{M}_{0, k} \cong S_{0, k}^{(0,0,1, \ldots, 1)}$.

Finally, by pull-back from the moduli space $\overline{\mathcal{M}}_{2,1}$, the one-dimensional group $H^{8}$ is tautological.

Finally, we compute the rational Chow ring of $\bar{S}_{g}^{ \pm}$for $g=3,4$ and show that the Picard group in this range is generated by the classes $\alpha^{ \pm}, \beta^{ \pm}$and $\mu$. For $g=2$ this has been worked out in [5]. 
Let $\mathcal{H} S_{g}^{ \pm}$be the preimage of the hyperelliptic locus $\mathcal{H}_{g}$. point.

Lemma 5.6. The space $\mathcal{H} S_{g}^{ \pm}$is affine and has the (rational) Chow ring of a

Proof. Since $\mathcal{H} S_{g}^{ \pm}$is the preimage of an affine set under a finite map, it is clearly affine. There exist maps

$$
\begin{aligned}
\phi^{+}: \mathcal{M}_{0,2 g+2} & \rightarrow \mathcal{H} S_{g}^{+}, \\
\phi^{-}: \mathcal{M}_{0,2 g+2} & \rightarrow \mathcal{H} S_{g}^{-} .
\end{aligned}
$$

The map $\phi^{+}$sends a rational pointed curve $\left[\mathbb{P}^{1} ; p_{1}, \ldots, p_{2 g+2}\right]$ in a hyperelliptic curve with with the theta characteristic $P_{1}+\cdots+P_{g+1}-g_{2}^{1}$, where the $g_{2}^{1}$ defines the hyperelliptic curve. The image point of $\phi^{-}$has theta characteristic $P_{1}+\cdots+P_{g-1}$. This is a surjective map, hence the Chow ring of $\mathcal{H} S_{g}$ is trivial since that of $\mathcal{M}_{0,2 g+2}$ is.

THEOREM 5.7. The moduli space $S_{3}^{-}$is the union of two affine spaces $\mathcal{H} S_{g}^{-}$and $W$. Each of them has trivial Chow ring, hence the rational Chow ring is $\mathbb{Q}$-spanned by the cycles $\left[\mathcal{H} S_{g}^{-}\right]$and $[W]$.

Proof. As proved in [18], the moduli space $\mathcal{M}_{3}$ is the union of two affine spaces, the hyperelliptic locus and its complement. Denote by $\mathcal{H} S_{g}^{-}$and $W$ the preimages under the forgetful map $S_{3} \rightarrow \mathcal{M}_{3}$. By the Lemma 5.5, the preimage of the hyperelliptic locus has trivial Chow ring. As proved in [18], the space $\mathcal{M}_{3}-\mathcal{H}_{3}$ is the image under a finite map $\Phi$ of a space with trivial Chow ring. The map is defined in such a way that it factorizes through the moduli space $S_{3}^{-}$. Hence $W$ has the Chow ring of a point.

Corollary 5.8. The ring $A^{\bullet}\left(S_{3}^{-}\right)$is isomorphic to the ring $\mathbb{Q}\left[\lambda^{-}\right] /\left(\left(\lambda^{-}\right)^{2}\right)$, where $\lambda^{-}$is the first Chern class of the Hodge bundle on $S_{3}^{-}$.

Proof. The map $\mathbb{Q}\left[\lambda^{-}\right] /\left(\left(\lambda^{-}\right)^{2}\right)$ is injective and by Theorem 5.7 is also surjective. Hence the claim follows.

Proposition 5.9. The (rational) Picard group of $\bar{S}_{3}^{-}$is generated by the classes $\alpha_{i}^{-}, \beta_{i}^{-}, \mu^{-}$.

Proof. By Theorem 3.6 in [11], the class $\mu^{-}$is a multiple of $\lambda^{-}$on $S_{3}^{-}$. Recall the exact sequence

$$
A_{5}\left(\partial S_{3}^{-}\right) \rightarrow A_{5}\left(\bar{S}_{3}^{-}\right) \rightarrow A_{5}\left(S_{3}^{-}\right) \rightarrow 0 .
$$

By the previous corollary, it follows that $A_{5}\left(S_{3}^{-}\right)$is generated by $\mu^{-}$, so $A_{5}\left(\bar{S}_{3}^{-}\right)$ is generated by boundary divisors and the class $\mu^{-}$.

Corollary 5.10. $\operatorname{Pic}_{\mathbb{Q}}\left(S_{3}^{-}\right) \cong \mathbb{Q}$.

An analogous statement holds in genus four.

TheOREm 5.11. The moduli space $S_{4}^{-}$admits a stratification by subvarieties whose successive differences are affine. Moreover, each of them has the Chow ring of a point. 
Proof. As proved in [18], Thm. 3.1, the moduli space $\mathcal{M}_{4}$ has a stratification $\mathcal{M}_{4} \supset \mathcal{M}_{4}^{\prime} \supset \mathcal{H}_{4}$, where $\mathcal{M}_{4}^{\prime}$ is the Thetanull divisor and $\mathcal{H}_{4}$ is the hyperelliptic locus. The successive differences are affine and have the Chow ring of a point. The preimages $S_{4}^{ \pm}, S_{4}^{ \pm^{\prime}}, \mathcal{H} S_{4}^{ \pm}$of the strata yield a stratification of $S_{4}^{-}$and $S_{4}^{+}$whose successive differences are affine.

The subvarieties $\mathcal{M}_{4}-\mathcal{M}_{4}^{\prime}$ and $\mathcal{M}_{4}^{\prime}-\mathcal{H}_{4}$ have the Chow ring of a point because there exists a finite morphism from the complement of a hypersurface $H$ in a projective space $\mathbb{P}$. Such a morphism factorizes through the moduli space of odd theta characteristics of genus four. Therefore, by Lemma 2.2. in [18], $S_{4}^{-}-{S_{4}^{-{ }^{\prime}}}^{\text {and }}$ $S_{4}^{-^{\prime}}-\mathcal{H} S_{4}^{-}$have the Chow ring of a point.

COROllary 5.12. The map

$$
\mathbb{Q}\left[\lambda^{-}\right]\left(\left(\lambda^{-}\right)^{3}\right) \rightarrow A_{\mathbb{Q}}^{\bullet}\left(S_{4}^{-}\right)
$$

is an isomorphism.

Proof. By Theorem 5.11, the Chow ring is spanned by the classes of $S_{4}^{-}, S_{4}^{-^{\prime}}$ and $\mathcal{H} S_{4}^{-}$. The class of $S_{4}^{-^{\prime}}$ is the pull-back of the class of the Thetanull divisor, hence a multiple of $\lambda^{-}$. Moreover, the class of $\mathcal{H} S_{4}^{-}$is a multiple of the class of the hyperelliptic locus, which is a multiple of $\lambda^{2}$. Therefore, the map 5.5 is injective and surjective.

Analogously to Proposition 5.9 and Corollary 5.10, and with the same proof, the following holds.

Proposition 5.13. i) The (rational) Picard group of $\bar{S}_{4}^{-}$is generated by $\mu^{-}$and the boundary classes $\alpha_{i}^{-}$and $\beta_{i}^{-}$.

ii) $\operatorname{Pic}_{\mathbb{Q}}\left(S_{4}^{-}\right) \cong \mathbb{Q}$.

6. Concluding Remarks. In Section 5 we showed that some Chow rings are tautological in low genus. Clearly, this work is only partial and we hope to come back on this in the next future. In particular, it would be interesting to study the whole structure of the Chow ring of the moduli space $\bar{S}_{2,1}$. Moreover, in all the other cases we have dealt with, it is an open question to find relations among the tautological classes.

In [6], we proved that $P i c_{\mathbb{Q}}\left(\bar{S}_{g}\right)$ is tautological for $g \geq 9$. In [5], we prove that the degree two cohomology group of $\bar{S}_{1, n}^{+}$is tautological. It is therefore reasonable to formulate the following

Question. $H^{2}\left(\bar{S}_{g, P} ; \mathbb{Q}\right) \cong P i c_{\mathbb{Q}}\left(\bar{S}_{g, P}\right)$ for $g \geq 0$.

Although expected, this result might be non-trivial to prove. The inductive techniques in [2] do not work directly because the boundary of the moduli space is made up by components which are not of the same type as $S_{g, n}$, but different spaces of square roots.

7. Acknowledgments. The author would like to thank Sergio Cacciatori, Roberto Catenacci, Francesco Dalla Piazza, Barbara Fantechi. The author is grateful to Claudio Fontanari for useful remarks and suggestions and to Filippo Viviani for showing him the argument in Lemma 5.6. This research was supported by the University of Milan "FIRST" and Cofin2006 - Varietà algebriche: geometria, aritmetica e strutture di Hodge. 


\section{REFERENCES}

[1] D. Abramovich and T. J. Jarvis, Moduli of twisted spin curves, Proc. Amer. Math., 131 (2003), pp. 685-699.

[2] E. Arbarello and M. Cornalba, Calculating cohomology groups of moduli spaces of curves via algebraic geometry, Inst. Hautes Études Sci. Publ. Math., 88 (1998), pp. 97-127 (1999).

[3] E. Arbarello and M. Cornalba, Combinatorial and algebro-geometric cohomology classes on the moduli spaces of curves, J. Algebraic Geom., 5:4 (1996), pp. 705-749.

[4] E. Arbarello and M. Cornalba, The Picard groups of the moduli spaces of curves, Topology, 26:2 (1987), pp. 153-171.

[5] G. Bini and C. Fontanari, On the geometry of $\bar{S}_{2}$, to appear in Intern. J. Math.

[6] G. Bini And C. Fontanari, Moduli of curves and spin structures via algebraic geometry, Trans. Amer. Math. Soc., 358 (2006), pp. 3207-3217.

[7] G. Bini, Generalized Hodge classes on the moduli space of curves, Beiträge zur Algebra und Geometrie, 44:2 (2004), pp. 559-565.

[8] G. Bini and C. Fontanari, A remark on the cohomology of $\bar{S}_{1, n}^{+}$, to appear in Coll. Math.

[9] P. Belorousski, Chow rings of moduli spaces of pointed elliptic curves, $\mathrm{Ph}$. D. dissertation, University of Illinois, 1998.

[10] R. Catenacci, M. Cornalba, M. Martellini and C. Reina, Algebraic Geometry and path integrals for closed strings, Phys. Lett. B, 172:3 (1986), pp. 328-332.

[11] M. CoRnalba, A remark on the Picard group of spin moduli space, Rend. Mat. Accad. Lincei, 2:9 (1991), pp. 211-217.

[12] M. CoRnalba, Moduli of curves and theta characteristics, Lectures on Riemann surfaces (Trieste, 1987), pp. 560-589, Teaneck, NJ: World Sci. Publishing, 1989.

[13] A. Chiodo, Stable twisted curves and their r-spin structures, Ann. Inst. Fourier (Grenoble), 58:5 (2008), pp. 1635-1689.

[14] A. Chiodo, Towards an enumerative geometry of the moduli space of twisted curves and $r$-th roots, Comp. Math., 144 (2008), pp. 1461-1496.

[15] D. Eisenbud AND J. HARRIS, The Kodaira dimension of the moduli space of curves of genus $\geq 23$, Invent. Math., 90 (1987), pp. 359-387.

[16] C. FABER, A conjectural description of the tautological ring of the moduli space of curves, In: Moduli of curves and abelian varieties, The Dutch Intercity Seminar on Moduli (C. Faber, E. Looijenga eds.) Aspects of Maths. E33, pp. 109-129, Vieweg 1999.

[17] C. FABER, Chow rings of moduli spaces of curves, Ph. D. dissertation, University of Amsterdam, 1988.

[18] C. Fontanari and E. Looijenga, A perfect stratification of $\mathcal{M}_{g}$ for $g$ at most 5, Geom. Dedicata, 136 (2008), pp. 133-143.

[19] J. Haren, The rational Picard group of the moduli space of Riemann surfaces with spin structure, Mapping class groups and moduli spaces of Riemann surfaces (Göttingen, 1991/Seattle, WA, 1991), pp. 107-136, Contemp. Math. 150, Amer. Math. Soc., Providence, RI, 1993.

[20] T. Jarvis, Geometry of the moduli of spin curves, Intern. J. Math., 11:5 (2000), pp. 637-663.

[21] T. Jarvis, T. Kimura And A. VaintroB, Moduli spaces of higher spin curves and integrable hierarchies, Comp. Math., 126 (2001), pp. 157-212.

[22] A. M. Polyakov, Quantum geometry of bosonic strings, Phys. Lett. B, 103:3 (1981), pp. 207210.

[23] A. Putman, The second rational homology group of the moduli space of curves with level structures, e-print arXiv:0809.4477.

[24] D. Mumford, Towards an enumerative geometry of the moduli space of curves, Arithmetic and Geometry (M. Artin and J. Tate, eds.) Part II, Birkhäuser 1983, pp. 271-328.

[25] D. Mumford, Stability of projective varieties, Enseign. Math. (2), 23:1-2 (1977), pp. 39-110.

[26] C. Volsin, Hodge theory and complex algebraic geometry, I Cambridge Studies in Advanced Mathematics, 76, Cambridge University Press, Cambridge, 2002. 